
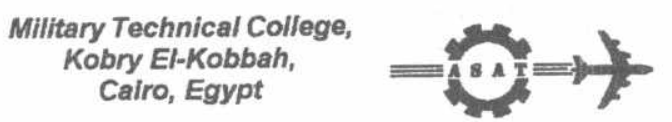

$9^{\text {th }}$ International Conference

On Aerospace Sciences \&

Aviation Technology

\title{
CO-CHANNEL INTERFERENCE CANCELLER FOR CELLULAR SYSTEMS
}

\author{
Soleit* E. A., El-Osmani* A., Ahmed* T. M.
}

\section{ABSTRACT}

This problem of the co-channel interference arises extensively in the cellular channels, which degrades significantly the performance of the cellular system. The interference signals are indeed the transmitted signals of the adjacent user in the neighboring zones. Then, the cancellation of these interference signals is of great interest to improve the system performance. However, the signal of interest is not known in advance. Hence, the classical method of noise cancellation is inefficient. The adaptive interference canceller (AIC) is introduced to cancel the co-channel interference signals.

A distributed AIC is proposed to cancel six interference signals. The proposed AIC has succeeded to cancel six interference sources and it exhibits a great improvement in the output signal to interference ratio.

\section{Introduction}

The co-channel interference results from the adjacent base stations. Output signals result in degrading the system performance and severely annoy the subscribers in the cellular network. Hence, the cancellation of this type of interference is necessary to improve the system performance and to make full use of the cellular network efficiency [1]. The classical methods for cancellation of these types of interference are inefficient; hence, the adaptive filtering technique is introduced to solve this problem. The adaptive noise cancellation technique is used, to get rid of the undesired interference signals that accompany the desired signal received from the assigned base station [2]. The direction of interference signals is estimated previously according to the space distribution of the cellular cells. Directive

\footnotetext{
*Egyptian Armed Forces
} 
Proceedings of the $9^{\text {th }}$ ASAT Conference, 8-10 May 2001 Paper AV-14 818

antennas that are directed towards the undesired stations, in the assigned zone collect the received signals from the undesired directions.

In this paper, a distributed adaptive filter configuration for the cellular system is presented. Each direction at antenna is used to receive the undesired signals from an assigned coverage direction; the output of each antenna sensor is applied to the corresponding adaptive filter. The filter outputs are summed and subtracted from the received signal from the primary channel that includes the desired signal combined with the interference signals [3]. The weight coefficients of the adaptive filter are updated using the well-known least mean square (LMS) adaptation algorithm such that the mean square of the resulting error signal is minimized $[5,6,7,8]$.

The distributed scheme is tested through the computer simulation for two and six speech channels. The simulation results are promising and a significant improvement in the output signal to interference ratio is obtained.

This paper includes five sections; section two explains the structure of the adaptive noise canceller. While section three presents the distributed noise canceller for the cellular system. Simulation results are presented in section four. Section five includes conclusion of the whole paper.

\section{II- The adaptive interference canceller}

An adaptive interference canceller is depicted in figure 1. The AIC includes two channels, the primary and the reference ones. The primary signal contains the desired signal in addition to the interference signal.

The primary signal is written as: -

$$
s(k)=x_{d}(k)+n_{i}(k)
$$

Where $n_{i}$ is highly correlated with the interference signal $X_{i}$. The reference signal $x_{r}$ is highly correlated with the interference signal.

The reference signal, $x_{r}$ can be expressed as: -

$$
x_{r}(k)=\sum_{m=0}^{N} h 1(m) x_{i}(k-m)
$$

Where $x_{i}$ is the interference signal .

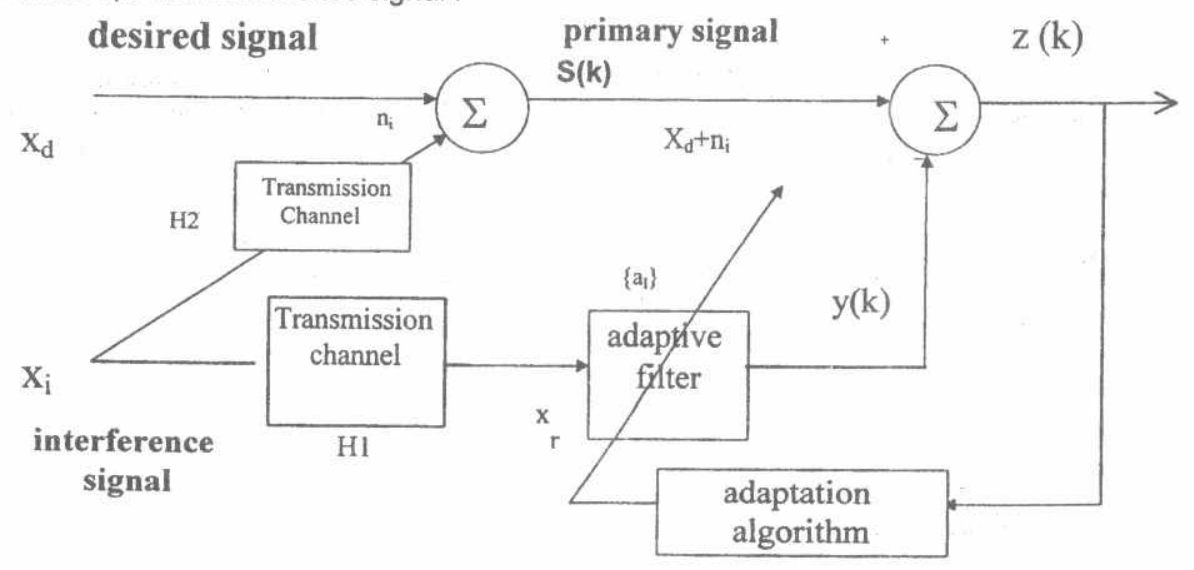

Fig. 1 An adaptive interference canceller 
The reference signal $x_{r}$ is introduced to an adaptive filter whose output, $y(k)$ is expressed by:

$$
y(k)=\sum_{i=0}^{M} a_{i}(k) x_{r}(k-i)
$$

The filter output is considered as a good estimate of the interference signal and hence, it is subtracted from the primary signal resulting an error signal, $z(k)$ as: $z(k)=s(k)-y(k)$

The weight coefficients $\left\{a_{i}\right\}$ are updated according to the well-known LMS adaptation algorithm such that, $E\left[z_{k}^{2}\right]$ is minimized $[3,5,6,7,8]$. Hence, the weight coefficients are updated as:

$a_{i}(k+1)=a_{i}(k)+2 \mu_{Z_{k}} x_{r}(k-i)$

Where $\mu$ is the step size of the adaptation algorithm that controls the adaptation speed and the steady state error.

\section{III- A distributed adaptive interference canceller (AIC)}

A distributed AIC is depicted in figure 2.It is used to cancel the co-channel interference in the cellular network.

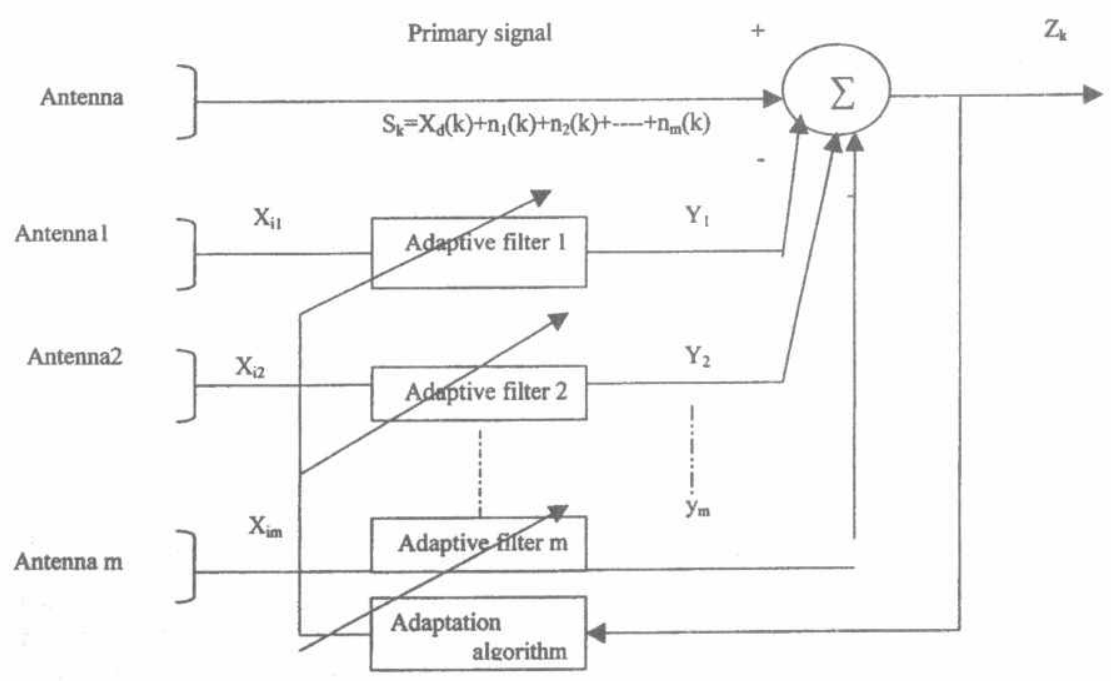

Fig 2 A distributed adaptive interference canceller 
The primary signal $\mathbf{s}(\mathbf{k})$ includes the desired signal in addition to the interference signals. In this case, the primary signal, $\mathrm{s}_{\mathrm{k}}$ can be expressed as [4]: -

$S_{k}=\boldsymbol{x}_{d}(k)+\sum_{j=1}^{L} n_{j}(k)$

Each filter output can be considered as a good estimate of an interference source and it subtracted from the primary signal resulting an error signal. $Z_{k}$ as:

$\mathbf{Z}_{\mathrm{k}}=\boldsymbol{S}_{k}-\sum_{j=1}^{L} \boldsymbol{y}_{j}(k)$

The filter output can be expressed as in eq.(3). Then, the filter coefficients are updated according to the LMS adaptation algorithm as:

$$
\begin{aligned}
& a_{l, i}(k+1)=a_{l, i}(k)+2 \mu z_{k} x_{r l}(k-i) \\
& i=\mathbf{1}, \mathbf{2}, \mathbf{3}, \ldots \ldots \ldots \ldots . . . ., M \\
& \boldsymbol{l}=\mathbf{1}, \mathbf{2}, \mathbf{M}, \ldots \ldots \ldots \ldots, \boldsymbol{L}
\end{aligned}
$$

Where $\left\{a_{1,1}\right\}$ are the filter coefficients of the distributed adaptive filters in the distributed interference canceller.

\section{IV-Simulation results}

The proposed distributed AIC depicted in Fig. 2 is tested through computer simulation to cancel the interference signals occurring in the cellular network. In this simulation, the implemented AIC is used to cancel six interference sources with 0.1 step size and 3 weight coefficients. The interference signal is represented by a real speech signal that is obtained from a voice card type (creative). Six speech waveforms are obtained from six independent speakers. 74000 samples of speech signals are used for simulation. Fig. 3 represents the desired speech signal. Fig. 4 illustrates the combined signal that includes the desired signal in addition to the interference ones .The six interference signals are presented in Fig. 5. Figure 6 demonstrates the output signal of the distributed AIC at the steady state after 5000 iterations. More over, Fig. 7 shows the learning curve of the distributed AIC.

It is clear that the distributed AIC has succeeded to cancel six interference sources with a great improvement in the output signal to interference ratio. 


\section{V-Conclusions}

The adaptive interference canceller (AIC) is introduced to cancel the co-channel interference signals. An adaptive interference canceller is performed through computer simulation. The distributed AIC proposed to cancel multi-interference signals, which has succeeded to cancel six interference signals. Simulation results have shown that the AIC provides significant improvement in the output signal to interference ratio.

\section{REFERENCES}

[1] Glover, J. R., Adaptive noise canceling applied to sinusoidal interference, IEEE Trans. Acoust. Speech, and Signal Processing, vol.ASSP-25,pp. 484-491. (1977).

[2] Haykin, S., Introduction to adaptive filters, Macmillan Pub. Co. (1984).

[3] Haykin, S., Adaptive filter theory, Prentice Hall Inc (1991).

[4] Soleit, E. A., The adaptive recursive speech enhancer, Al-Azhar Engineering International Conference AEIC, vol. 6, pp. 217-227, December (1997).

[5] W.C.Y. Lee, mobile cellular telecommunication systems. New York: McGraw-Hill, 1989

[6] Widrow, B., and Stearns, S. D., Adaptive signal processing Prentice Hall Inc. (1985).

[7] Widrow, B., et al., Adaptive noise canceling principles and applications, PROC. IEEE, vol. 63, pp. 1692-1716, December (1975).

[8] Pi sheng chang and Alan N. Wilson, Analysis of conjugate Gradient Algorithms for adaptive filtering Proc. IEEE. (2000) 


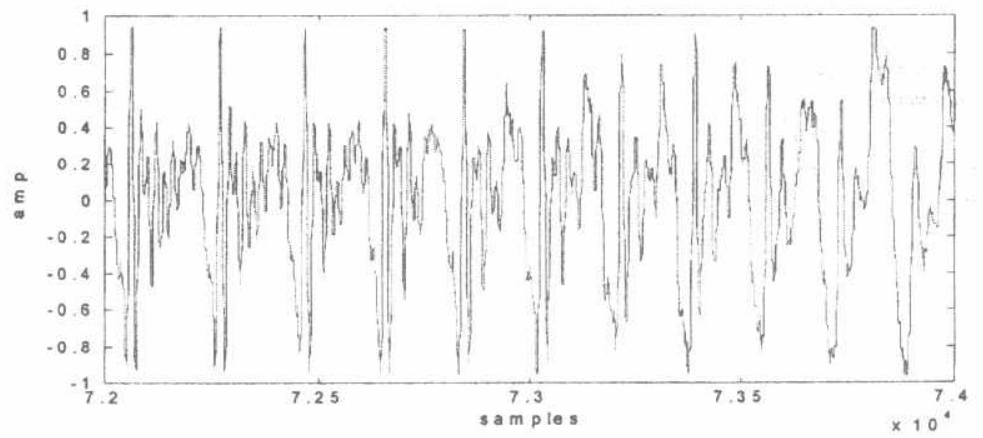

Fig.3 Desired speech signal (110KB)

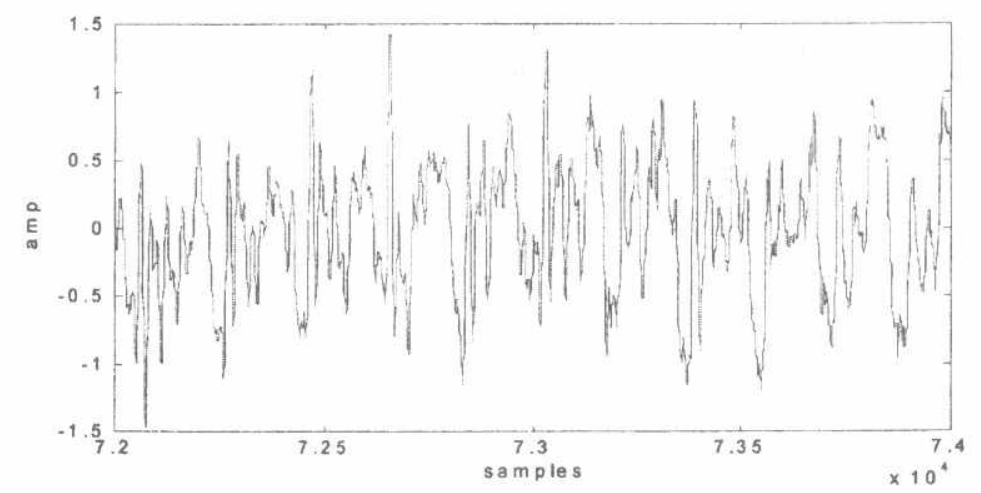

Fig.4 The combined signal (desired signal + six interference signals) 


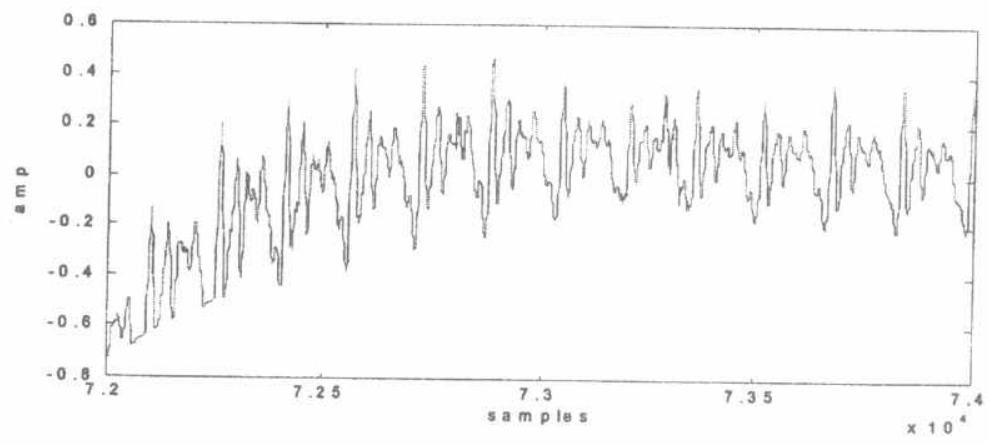

(a) Interference 1 (75.3 KB)

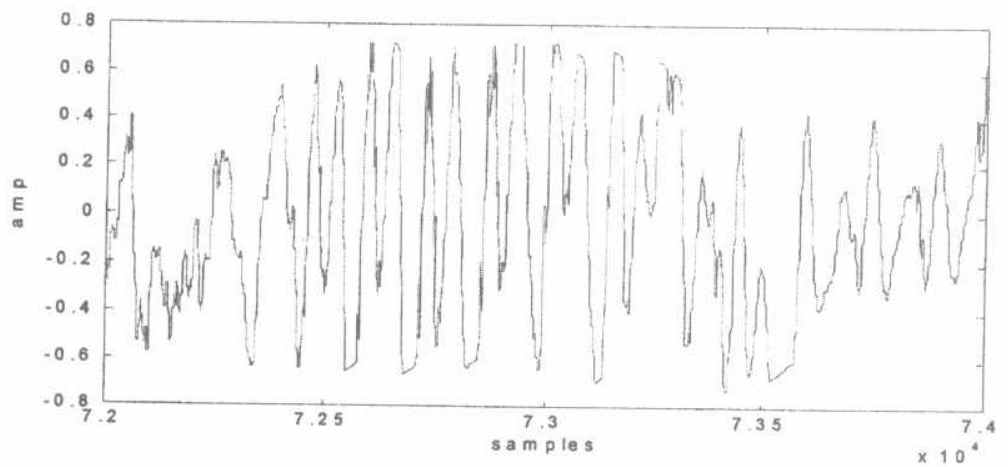

(b) Inteference 2 (146 KB)

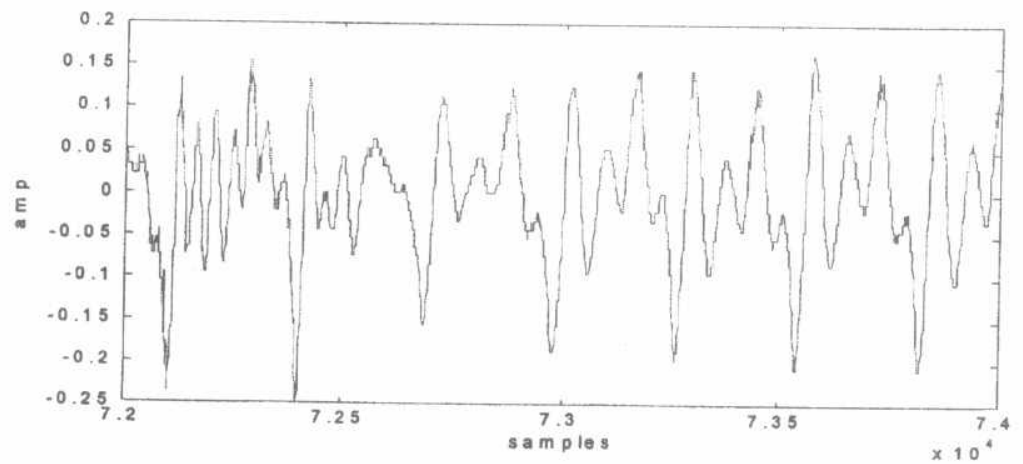

(c) Interference 3 (130KB) 


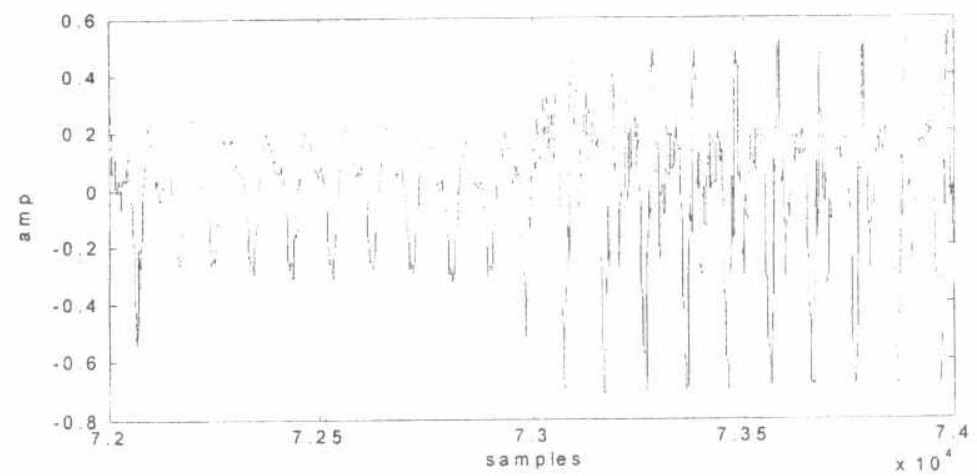

(d) Interference 4 (131 KB)

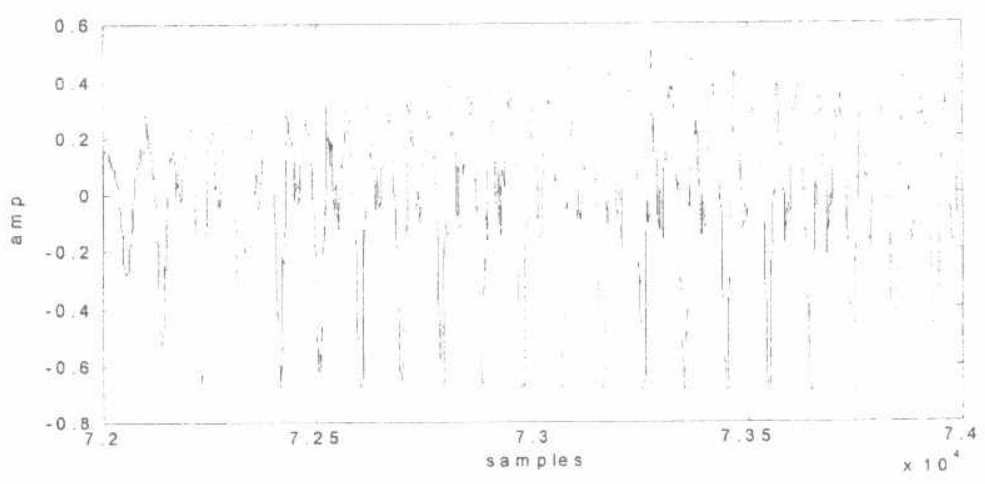

(e ) Interference $5(116 \mathrm{~KB})$

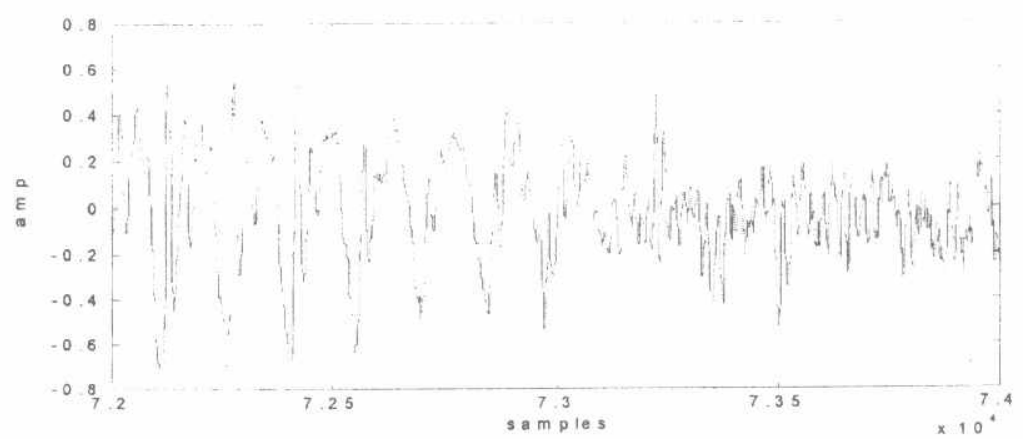

(f) Interference 6 (104 KB)

Fig. 5 six interference signals (a), (b), (c), (d), (e), (f) from 72000 to 74000 samples. 


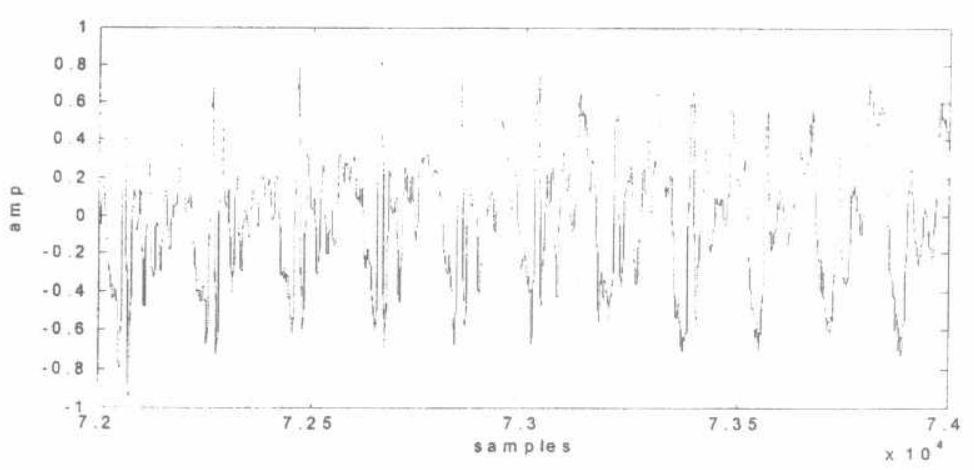

Fig. 6 The output signal of the distributed AIC $(M=3, L=6$, ISIR $=-5 \mathrm{~dB}$, output $\mathrm{SIR}=17.2 \mathrm{~dB}$ and Improvement $=22.2 \mathrm{~dB}$ )

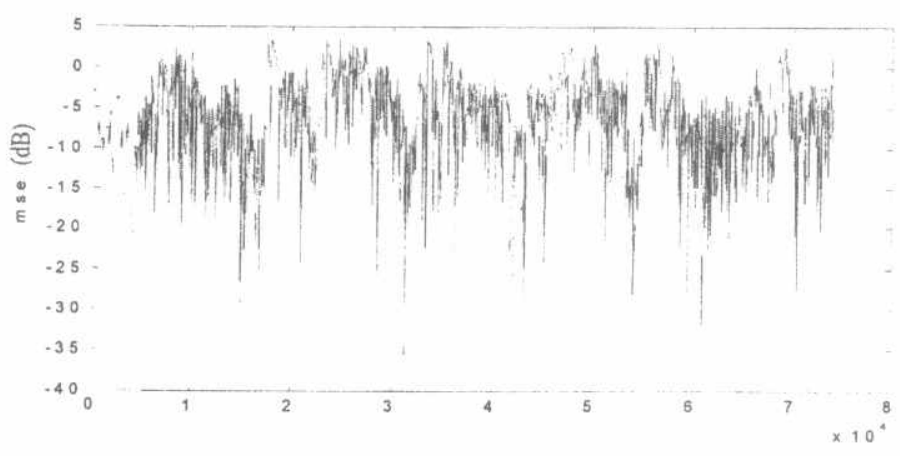

Fig.7 The learning curve of the distributed AIC $(M=3, L=6, I S I R=-5 d B$, output $\mathrm{SIR}=17.2 \mathrm{~dB}$ and Improvement $=22.2 \mathrm{~dB}$ ) 